\title{
Alcohol-Related Liver Disease in the Covid-19 Era: Position Paper of the Italian Society on Alcohol (SIA)
}

\author{
Gianni Testino ${ }^{1} \cdot$ Teo Vignoli $^{2} \cdot$ Valentino Patussi $^{3} \cdot$ Pierluigi Allosio $^{4} \cdot$ Maria Francesca Amendola $^{5} \cdot$ Sarino Arico ${ }^{6}$. \\ Aniello Baselice ${ }^{7} \cdot$ Patrizia Balbinot $^{1} \cdot$ Vito Campanile $^{8} \cdot$ Tiziana Fanucchi $^{3} \cdot$ Giovanni Greco $^{9} \cdot$ Livia Macciò $^{10}$. \\ Cristina Meneguzzi ${ }^{11}$. Davide Mioni ${ }^{12} \cdot$ Vincenzo Ostilio Palmieri $^{13} \cdot$ Michele Parisi $^{14} \cdot$ Doda Renzetti $^{15}$. \\ Raffaella Rossin ${ }^{16}$. Claudia Gandin ${ }^{17}$. Luigi Carlo Bottaro ${ }^{18}$. Mauro Bernardi ${ }^{19}$. Giovanni Addolorato ${ }^{20}$. \\ Lisa Lungaro ${ }^{21}$. Giorgio Zoli ${ }^{21,22,23}$. Emanuele Scafato ${ }^{17} \cdot$ Fabio Caputo $^{21,22,23}$ (1)
}

Received: 20 July 2020 / Accepted: 14 April 2021 / Published online: 17 June 2021

(c) The Author(s), under exclusive licence to Springer Science+Business Media, LLC, part of Springer Nature 2021

\begin{abstract}
Background Coronavirus Disease 2019 (COVID-19), firstly reported in China last November 2019, became a global pandemic. It has been shown that periods of isolation may induce a spike in alcohol use disorder (AUD). In addition, alcoholrelated liver disease (ALD) is the most common consequence of excessive alcohol consumption worldwide. Moreover, liver impairment has also been reported as a common manifestation of COVID-19.

Aims The aim of our position paper was to consider some critical issues regarding the management of ALD in patients with AUD in the era of COVID-19.

Methods A panel of experts of the Italian Society of Alcohology (SIA) met via "conference calls" during the lockdown period to draft the SIA's criteria for the management of ALD in patients with COVID-19 as follows: (a) liver injury in patients with ALD and COVID-19 infection; (b) toxicity to the liver of the drugs currently tested to treat COVID-19 and the pharmacological interaction between medications used to treat AUD and to treat COVID-19; (c) reorganization of the management of compensated and decompensated ALD and liver transplantation in the COVID-19 era.

Results and Conclusions The COVID-19 pandemic has rapidly carried us toward a new governance scenario of AUD and ALD which necessarily requires an in-depth review of the management of these diseases with a new safe approach (management of out-patients and in-patients following new rules of safety, telemedicine, telehealth, call meetings with clinicians, nurses, patients, and caregivers) without losing the therapeutic efficacy of multidisciplinary treatment.
\end{abstract}

Keywords Alcohol-related liver disease · Alcohol-related liver cirrhosis · Alcohol use disorder - SARS-CoV-2 infection . Management of AUD · ALD and SARS-CoV-2

\section{Introduction}

Severe Acute Respiratory Syndrome (SARS) due to Coronavirus 2 (CoV-2) and Coronavirus Disease 2019 (COVID-19) was first reported in Wuhan (China) in November 2019 [1, 2], and by March 2020, the World Health Organization had declared a global pandemic. It is the cause of a worldwide pandemic affecting more than 200 countries. Most governments have responded to the COVID-19 pandemic by imposing a lockdown to save lives and to protect themselves

Fabio Caputo

fabio.caputo@unife.it

Extended author information available on the last page of the article and their healthcare systems. By February 14, 2021, more than 100 million people worldwide had been infected with SARS-CoV-2 [3]. Italy was one of the countries worst hit by the spread of this highly infectious disease. On February 14, there were 2.72 million infected subjects in Italy, 2.23 million of whom recovered, while 93,577 died; even though the number of infected subjects is decreasing and the vaccination program is fully active, the number of deaths per day continues to be high (usually about 300 subjects), and the recent finding of the English variant in Italy leads to think that it will be a long time before the pandemic is over.

Alcohol consumption worldwide is responsible for approximately $5.9 \%$ of all deaths (3.3 million deaths) and accounts for $5.1 \%$ of the global disease burden $[4,5]$. In 
addition, alcohol consumption can lead to approximately 200 different diseases, including fourteen different types of cancer, a greater susceptibility to infections $[4,5]$, and can also have an addictive element [6]. Moreover, the worldwide prevalence of alcohol use disorder (AUD) ranges between 3 and $15 \%[7,8]$. In addition, some evidence suggests that the period of isolation might have led to a spike in alcohol abuse [9]. Indeed, a recent study performed in China during the period of lockdown for COVID-19 showed that hazardous and harmful drinking both increased greatly from $4.4 \%$ to $11.1 \%$ [10].

Alcohol-related liver disease (ALD) together with AUD is the most common consequences of excessive alcohol consumption worldwide [11, 12]. In addition, alcohol-related liver cirrhosis (ALC) is responsible for $0.9 \%$ of global deaths and $47.9 \%$ of cirrhosis-related deaths, and it is expected that ALD will become the main indication for liver transplantation (LT) along with cirrhosis due to nonalcoholic steatohepatitis $[12,13]$.

Liver impairment has also been reported as a common manifestation of COVID-19, although its clinical significance is still unclear; however, it is important to define whether ALD should be considered a risk factor for a severe disease course of COVID-19. Thus, a better awareness in the management of ALD in patients affected by COVID-19 will be an essential part of the clinical skills of specialists in gastroenterology, hepatology, internal medicine, and addictive disorders who will encounter patients with this complex condition in their daily clinical practice.

\section{Methods}

A panel of clinicians, psychologists, and social-health professionals consisting of specialists in gastroenterology, internal medicine, gerontology, clinical pharmacology, psychiatry, toxicology experts in the treatment of AUD and ALD, and psychotherapists was identified as appropriate by the board of the Italian Society of Alcohology (SIA) and met via "conference calls" and other technological forms of meeting during the lockdown period (from March 2 to May 8,2020 ) to draft the ISA's criteria for the management of ALD in patients with COVID-19. On the grounds of their competence, role, expertise, and publications in the field of ALD and AUD, twenty of them were chosen to collect and finalize the draft and its recommendations. From September 1 to December 15, 2020, further meetings were planned in order to revise the paper with the participation of two Italian international experts in the field of AUD and ALD outside SIA's affiliation. The final paper then underwent revision and approval by the ISA board. This discussion lasted until January 15, 2021.
The aim of our position paper was to consider some critical issues regarding the management of ALD in the era of COVID-19. In our opinion, the most significant topics to be discussed were: (a) liver injury in patients with ALD and COVID-19 infection; (b) toxicity to the liver of the drugs currently tested to treat COVID-19 and the pharmacological interaction between medications used to treat AUD and to treat COVID-19; (c) reorganization of the management of compensated and decompensated ALD together with LT in the COVID-19 era. The data used to prepare the position paper were based on the analysis of the scientific literature published between March 1, 2020, and May 15, 2020. In particular, in the development process of this position paper we consulted the indications of the European Association for the Study of the Liver (EASL), the European Society of Clinical Microbiology and Infectious Diseases (ESCMID) $[14,15]$, the American Association for the Study of the Liver (AASL) [16], the Asian-Pacific Society for the Study of the Liver (APASL) [17], the World Gastroenterology Organization (WGO) [18], the European Centre for Disease Prevention and Control (ECDPC) [19], and those of the World Health Organization (WHO) with their continuous updates on COVID-19 [20].

Thus, the position paper was produced with the intent of providing specialists in gastroenterology, hepatology, internal medicine, alcohol addiction, and psychopharmacology with appropriate tools to better manage patients with ALD, AUD, and COVID-19. However, it was not possible to identify any degrees and levels of evidence for the final recommendations due to the lack of scientific papers published regarding ALD, AUD, and COVID-19.

\section{Coronavirus Disease 19 (Covid-19)}

Last December 2019 in Wuhan (Hubei Province, China), several cases of pneumonia inducing a SARS were identified $[1,2]$, and the Chinese Centre for Disease Control of Prevention subsequently attributed the pneumonia to COVID-19 [21]. Other coronavirus species causing human infection had already been identified; namely, the Middle East Respiratory Syndrome Coronavirus (MERS) and SARS-CoV-1 [21, 22]. SARS-CoV-2 and its clinical manifestation called COVID19 are the latest one to be identified. COVID-19 receptor sites present a strong interaction with angiotensin 2-converting enzyme (ACE2) receptors mainly present in the lung, gut, kidney, and liver sites [23]. This results in easier penetration and diffusion, particularly in the lower respiratory tract; indeed, ACE2 is mainly expressed in type 2 alveolar cells. Human-to-human transmission is possible not only via the respiratory route, but also via the fecal-oral route [24, 25]. In addition, like other infections, this viral infection is responsible for the so-called hyper-inflammatory syndrome 
characterized by a pro-inflammatory molecule (cytokine) storm with multi-organ failure and increased mortality rates [26].

The most frequent symptoms of COVID-19 are: fever $(90 \%)$, dry cough $(82.6 \%)$, asthenia with headache and myalgia (70\%), and dyspnea (50\%). Even less frequent, gastroenterological signs and symptoms such as diarrhea have also been described [2, 23, 27]. The progression of the COVID19 disease is characterized by bilateral interstitial pneumonia complicated by acute respiratory distress syndrome and acute heart and kidney complications [23]. Laboratory findings typically include lymphopenia with leukocytosis, elevated lactate dehydrogenase (LDH), C-reactive protein (CRP), ferritin, and aminotransferases [23, 25, 27].

The WHO recommends that all patients with suspected SARS-CoV-2 infection should undergo a nasopharyngeal/ pharyngeal swab in laboratories authorized to perform nucleic acid amplification diagnostic tests: real-time RTPCR. This test has proven to be the most sensitive and specific tool [28].

\section{Alcohol-Related Liver Disease and Covid-19}

Almost $90 \%$ of subjects consuming $>40-60 \mathrm{~g} /$ day of alcohol may develop steatosis of the liver, 20-40\% an alcohol-related steato-hepatitis, and 8-20\% ALC. In addition, $20-40 \%$ of alcohol-related steato-hepatitis cases may lead to ALC, and $2-4 \%$ to hepatocellular carcinoma (HCC). Severe alcohol-related steato-hepatitis can lead to acute alcoholic hepatitis (AAH) which is considered a distinct clinical entity [29-31]. Regression of steatosis is possible after 4-6 weeks of complete abstinence from alcohol; despite the complete abstinence from alcohol, several studies have shown that the evolution in fibrosis and cirrhosis may occur in 5-15\% of steatosis (or steato-hepatitis) [29, 31-33]. Another clinical condition in ALD is the acute on chronic liver failure (ACLF) which may occur in 10-30\% of hospitalized patients [34]. According to the EASL Clinical Practice Guidelines, there is agreement that ACLF is a distinct syndrome, and it is defined by a condition in patients with underlying chronic liver disease with or without cirrhosis with multi-organ failure and higher short-term mortality than a "simple decompensation" of cirrhosis [35].

The ACE2 receptors are highly expressed in cholangiocytes, the level being about 20 times higher than that in hepatocytes [36]. Since cholangiocytes are the liver cells into which SARS-CoV-2 could actually enter, no case of severe acute hepatitis due to SARS-CoV-2 has been described yet; indeed, hepatocytes have a low/ intermediate baseline expression of ACE-2. Although alcohol liver damage "per se" increases the ACE2 expression, as demonstrated by Okuno et al. [37] particularly in cholangiocytes [38] of ALD patients, this was not subsequently confirmed. Therefore, besides liver damage potentially induced by COVID-19 via ACE2 receptors, other factors are involved in liver toxicity. First, hepatic dysfunction may result from a cytokine storm rather than the direct cytopathic effects of the virus: the release of numerous inflammatory cytokines induces adult respiratory distress syndrome (ARDS) and systemic inflammatory response syndrome (SIRS) and subsequently causes hypoxia in the body, thereby leading to an injury in the liver. Second, ARDS, SIRS, and multi-organ failure (MOF) may cause hepatic ischemia and hypoxia reperfusion dysfunction [39]. Third, drug toxicity may contribute to liver damage in severe and critical COVID-19 patients. Namely, compared with patients experiencing a mild or moderate clinical course, severe and critical patients require longer duration of antiviral therapy and multiple drugs combined: It has been reported that antiviral medications, antipyretics, and antibiotics can cause liver damage [39]. In addition, COVID-19 patients with pre-existing liver diseases may be more prone to have abnormal liver biochemical indicators [39]. Recent studies showed that the incidence of liver injury is higher (14-53\%) in patients with COVID19 , particularly in those with severe disease $[38,40]$.

The laboratory findings of liver damage during SARS$\mathrm{CoV}-2$ infection are expressed by the increase in AST, ALT, and GGT, while the onset of liver impairment correlates with a more serious prognosis [27, 40]. More focused reports have suggested that ALD makes up about $30 \%$ of hospitalized patients with COVID-19 [41]. Moreover, a recent meta-analysis showed that, at hospital admission, among the liver biochemical indicators, abnormal albumin was the most common, followed by GGT, AST, ALT, TBIL, and $\operatorname{ALP}(39.8 \%, 35.8 \%, 21.8 \%, 20.4 \%, 8.8 \%$, and $4.7 \%$ ), while, during hospitalization, abnormal ALT was more common than AST and TBIL $(38.4 \%, 28.1 \%$, and $23.2 \%)$. In addition, among the risk factors, severe and/or critical patients (admission to the intensive care unit, and to mechanical ventilation) had a significantly higher pooled incidence of abnormal liver biochemical indicators at admission than mild and/or moderate patients, and that nonsurvivors had a significantly higher incidence of abnormal liver biochemical indicators than survivors $(\mathrm{RR}=1.34, p=0.04)$. Thus, the authors concluded that abnormal liver biochemical tests are common in COVID-19 patients, and liver biochemical indicators are closely related to the severity and prognosis of COVID-19 patients [42]. Indeed, liver disease was recently included by the Centers for Disease Control and Prevention as a co-morbidity that places patients at a high risk of COVID-19. Therefore, the American Association for the Study of Liver Diseases (AASLD) recommends that these patients should be given priority for $\mathrm{CoV}-2$ testing if they manifest symptoms [43, 44]. 


\section{Medications and Liver Toxicity}

\section{Medication for the Treatment of COVID-19}

Many drugs, mainly those used for anti-viral therapy, and hydroxychloroquine, are under investigation to treat COVID19 [28, 45-48]. A recent finding showed that remdesivir, hydroxychloroquine, lopinavir, and interferon regimens had little or no effect on hospitalized patients with COVID-19, as indicated by overall mortality, initiation of ventilation, and duration of hospital stay [49]. However, remdesivir is the only drug approved by the FDA [50] and in Italy [51] for the treatment of COVID-19. On the other hand, corticosteroids (dexamethasone) are currently considered the only drug efficient in reducing mortality in patients with severe COVID19 disease needing oxygen support [52], and the use of low molecular weight heparin seems promising in hospitalized COVID-19 patients, even though the measurement of the real efficacy is still ongoing [53].

However, all these drugs may be hepatotoxic, particularly in patients with underlying chronic liver disease. In addition, it is worth noting that close monitoring of drug interactions in patients following an immunosuppressive therapy should be carried out. Lopinavir/ritonavir, an antiretroviral protease inhibitor, can cause transient and usually asymptomatic elevations in AST and ALT levels [54]. Hydroxychloroquine, an antimalarial agent, has not been associated with liver abnormalities, it rarely causes a clinically apparent acute liver injury, and adjustments of dose are not necessary in patients with liver impairment [55]. However, hydroxychloroquine should be used with caution since there continues to be no high-quality clinical data showing a clear benefit of these agents for COVID-19, and hydroxychloroquine has the potential to cause harm, including serious cardiac side effects such as prolongation of QT and cardiac arrhythmias [56]. Tocilizumab, an interleukin-6 inhibitor, frequently causes mild serum elevations of AST and ALT and bilirubin levels [57]. Ivermectin, an anti-parasitic agent, has been associated with minor, self-limiting serum aminotransferase elevations and very rare episodes of clinically detected liver injury [58]. Remdesivir is a novel nucleotide analog and may induce elevations of transaminase levels in about $20 \%$ of patients [59], and similarly, favipiravir, an RNA polymerase inhibitor, may also cause liver cytolysis [60].

\section{Medications for the Treatment of AUD}

At present, the following medications are approved for the treatment of AUD in different countries: disulfiram, naltrexone, nalmefene, acamprosate, sodium oxybate (SO), and baclofen [61, 62] (Table 1). However, sodium oxybate is approved only in Italy and Austria, and baclofen received a "temporary recommendation for use" in France. In other countries, these two drugs are used off-label.

Table 1 Medications currently approved in the USA and in some European countries for the treatment of alcohol use disorder and their possible use in patients with ALC

\begin{tabular}{|c|c|c|c|}
\hline Medication & Dosage & Mechanism of action & Use in ALC \\
\hline Acamprosate* & $\begin{array}{l}666 \mathrm{mg} \text { TID }(\geq 60 \mathrm{~kg}) \\
333 \mathrm{mg} \text { TID }(<60 \mathrm{~kg})\end{array}$ & Possibly NMDA receptor agonist & $\begin{array}{l}\text { Yes (no hepatic metabolism; renal metabolism); no } \\
\text { controlled trials }\end{array}$ \\
\hline Disulfiram* & $250-500 \mathrm{mg}$ QD & $\begin{array}{l}\text { Inhibition of acetaldehyde } \\
\text { dehydrogenase }\end{array}$ & $\begin{array}{l}\text { No (hepatic metabolism; cases of liver toxicity have } \\
\text { been reported); no controlled trials }\end{array}$ \\
\hline Naltrexone* & $\begin{array}{l}\text { PO or IM } \\
\text { PO: } 50 \mathrm{mg} \text { QD } \\
\text { IM: } 380 \mathrm{mg} \text { monthly }\end{array}$ & $\mu$ opiate receptor antagonist & $\begin{array}{l}\text { With caution (hepatic metabolism, possible liver toxic- } \\
\text { ity limit use in advanced alcoholic liver disease); no } \\
\text { controlled trials }\end{array}$ \\
\hline Nalmefene $^{\wedge}$ & $20 \mathrm{mg}$ "as needed" & $\begin{array}{l}\mu \text { and } \delta \text {-opioid receptor antagonist } \\
\text { and } \kappa \text {-opioid receptor partial- } \\
\text { agonist }\end{array}$ & $\begin{array}{l}\text { With caution (hepatic metabolism, possible liver toxic- } \\
\text { ity limit use in advanced alcoholic liver disease); no } \\
\text { controlled trials }\end{array}$ \\
\hline Baclofen $^{\circ}$ & 10 mg TID (80 mg QD max) & $\mathrm{GABA}_{\mathrm{B}}$ receptor agonist & $\begin{array}{l}\text { Yes (15\% hepatic metabolism; } 85 \% \text { renal metabolism); } \\
\text { performed trials present }\end{array}$ \\
\hline Sodium Oxybate§ & $\begin{array}{l}50-100 \mathrm{mg} / \mathrm{kg} \text { fractioned } \\
\text { into three or six daily } \\
\text { dosages }\end{array}$ & $\mathrm{GABA}_{\mathrm{B}}$ receptor agonist & $\begin{array}{l}\text { Possible (hepatic metabolism, very short half-life and } \\
\text { fractioned dose of administration may avoid accumu- } \\
\text { lation of the drug in impaired liver function); only } \\
\text { one case report, no controlled trials }\end{array}$ \\
\hline
\end{tabular}

\footnotetext{
*Medications approved by the US Food and Drug Administration (FDA) relapse prevention (abstinence)

${ }^{\wedge}$ Medication approved by the European Medical Agency (EMA) in some European countries for the reduction of alcohol consumption

${ }^{\circ}$ Medication approved by the France Agence Nationale du Sècuritè du Mèdicament ed des Produits de Santè (ANSM) as a temporary recommendation for use to treat alcohol dependence

${ }^{\S}$ Medication approved in Italy and Austria for the treatment of alcohol withdrawal syndrome and for the maintenance of alcohol abstinence
} 
Table 2 General indications for the daily clinical activity in hepatology centers (HCs) to manage patients with ALD/ALC and AUD in the COVID-19 era

Re-organization of the HCs (follow hygiene rules, use PPE, and social distancing)

Use telemedicine/telehealth (e-mail, telephone, or video call) to manage therapeutic alliance, to maintain clinical activity, and psychological intervention, and to support caregivers (i.e., avoid hospitalization due to hepatic encephalopathy, preferring at-home management in accordance with the caregiver)

Outpatient visits only for urgent and severe clinical cases, limiting out-patient visits to those with ALD and high MELD (score > 20) or patients who resumed heavy alcohol consumption in order to plan a new detoxification program

Treatment for ALC complications (portal hypertension, ascites, hepatic encephalopathy, spontaneous bacterial peritonitis, and gastrointestinal bleeding) should be continued (when it is possible such as for paracentesis, in a day hospital setting)

In the case of suspected CoV2 infection, request a nasopharyngeal/pharyngeal swab check

If a patient needs hospitalization for detoxification from alcohol, or for the treatment of a severe alcohol withdrawal syndrome, or in case of decompensation of ALC, this may be done in a non-COVID ward after SARS-CoV-2 testing

Disulfiram has been approved in the USA by the Food and Drug Administration (FDA) for the treatment of AUD since 1948. Its main mechanism of action is represented by the inhibition of aldehyde dehydrogenase, preventing the metabolism of alcohol's primary metabolite, acetaldehyde. It is mandatory to inform patients to avoid alcohol during treatment with disulfiram [63].

Naltrexone was approved in the USA by FDA in 1994 for the treatment of AUD. Naltrexone is a $\mu$ - and k-opioid receptor antagonist, used to reduce reward craving. Alcohol intake does not significantly interfere with treatment [61, 62].

Nalmefene is a $\mu$ - and $\delta$-opioid antagonist and k-opioid partial agonist, with long plasma half-life and high bioavailability. It is effective in reducing alcohol intake, in particular in reducing heavy drinking, at the dose of $18 \mathrm{mg} /$ day [64]. With this indication, it was recently approved in Europe by the European Medicines Agency.

Acamprosate was approved in the USA by the FDA in 2004 for the treatment of alcohol dependence. Acamprosate acts on N-methyl-d-aspartic acid receptors and metabotropic glutamate receptors [65, 66]. This medication reduces hyperglutamatergic activity and glutamate levels in AUD [67, 68]. It has no interaction with alcohol.

SO has been approved in Italy since 1992 and in Austria since 1999 for the treatment of AUD. This drug is a GABAreceptors agonist [69]. SO has an alcohol-mimicking effect due to an increased dopamine release mediated by $\mathrm{GABA}^{-}$ receptors.

Baclofen is a $\mathrm{GABA}_{-}{ }_{\mathrm{B}}$ receptor agonist, acting like an alcohol substitute on the reduction of alcohol reinforcement and reward [61]. In France, a "temporary recommendation for use" has been endorsed by the National Security Agency of Medicine and Health Products (MSNA) since 2014. Adverse effects are exacerbated by concurrent alcohol consumption [61].

In addition, psychiatric comorbidity is frequent in patients with AUD. Alcohol is the psychoactive substance most frequently used by patients with psychiatric disease. Some $40-70 \%$ of patients with AUD have a psychiatric comorbidity, and they also follow a specific pharmacological treatment with psychoactive drugs [7, 70].

All the pharmacological therapies used to treat COVID19 may negatively interfere with drugs currently used to treat patients with AUD and psychiatric co-morbidity. Hydroxychloroquine may potentially induce an increase in liver toxicity and neuropathy when used with disulfiram, and may increase the risk for the onset of diarrhea with acamprosate $[28,46,71,72]$. Thus, in the case of COVID-19 therapy with experimental drugs $[28,46,71,72]$, due to the severity of symptoms, the discontinuation of adversative or anti-craving drugs needs to be carefully evaluated. Hydroxychloroquine may cause the lengthening of the QT interval $[73,74]$, and on the other hand, alterations in the QT interval are also caused by psychotropic drugs (tricyclic antidepressants, antipsychotics, monoamino oxidase inhibitors, antiepileptics, bupropion, and others) [75].

\section{General Considerations}

Monitoring of drug-drug interactions in patients with AUD and a psychiatric comorbidity under multi-drug treatment needs to be planned [72]. Further precautions are, thus, warranted in patients with AUD who need anti-viral or antiinflammatory treatment for COVID-19, with the aim of discontinuing anti-craving or adversative drugs in the case of severe side effects. Specifically, some authors recommend the following cutoffs for immediate suspension: ALT values 3 or 5 times greater than the normal level and/or bilirubin levels beyond the normal limits [76, 77].

\section{Management of ALD/AUD in the Covid-19 Disease Era}

All healthcare systems need to be reorganized during and after the SARS-CoV-2 pandemic, as do Hepatology Centers (HCs) in order to combine operator safety with patient safety and care. HCs need essential equipment to carry out their 
clinical activities (Table 2). Some general rules need to be followed during daily activities in HCs.

\section{Telemedicine/Telehealth}

Telemedicine is the remote delivery of healthcare services using the Internet. Some people refer to it as "telehealth," or "mhealth" (short for "mobile health"). Video technology (like Skype, video calls) also enables people to report their problems to a doctor. Telemedicine has a wide range of applications. It can be used for counseling, chronic disease monitoring and management, professional education, and more. With modern telecommunications, a patient can call a doctor, describe his or her symptoms, and receive a diagnosis and a prescription in just a few minutes. Telemedicine enhances convenience, reduces travel time and costs, and offers additional benefits for patients, physicians, and the greater healthcare system. In addition, telemedicine may allow patients with AUD and ALD to stay in treatment and to receive counseling, reinforcing complete abstinence from alcohol or avoiding relapse. It has been demonstrated that telemedicine or telehealth can be a useful tool for patients with addiction [78] and allow a closer surveillance of patients at risk of liver decompensation and relapse [15, $79,80]$. Indeed, a recent pilot study has shown that specialized hepatology care can be effectively delivered through telehealth to distant communities not formerly serviced by transplant and HCs [81]. Despite these advantages, however, telehealth is constrained by Internet access, especially in rural areas [82] where many patients do not have computers or smartphones and lack access to telehealth video visits, so providers should utilize telephone visits when possible [83]. Therefore, in a period of social distancing, this form of support for patients with AUD and ALD needs to be implemented, and improving telemedicine services will have a significant long-term positive impact on patient care that will persist even after this pandemic [44].

\section{Out-Patient Treatment}

Access to an $\mathrm{HC}$ needs to be regulated to only one patient at a time in order to avoid gatherings in the waiting room. Signs must be posted on the access pathways to the HC and in the waiting room that illustrate the procedures adopted and reiterate the need to comply with the rules of conduct suggested by the Ministry of Health. The waiting rooms need to be reorganized with a limited number of seats which must be at a safe distance from each other as required by law (at least one meter apart in Italy) [84]. Patients are asked whether they have symptoms (i.e., fever, cough), and their temperature is detected with a thermo-scanner. If a patient has a body temperature $\geq 37.5 \mathrm{C}^{\circ}$, he/she is invited to go home and call his/her doctor and/or the dedicated facilities indicated by the health authorities. Moreover, during visits, health workers need to wear personal protective equipment (PPE) (surgical mask and gloves) and take care of hand hygiene before and after each intervention is performed. PPE must be replaced if it gets wet, and gloves must always be removed (properly) at the end of each operation and replaced after hand hygiene. Goggles/visors are reusable after sanitization with a chlorine-based product.

In addition, HCs must have contact with a reference caregiver to better manage therapeutic adherence at home in order to avoid inpatient care. In fact, $78 \%$ of hospital readmissions of patients with decompensated cirrhosis were totally or partially due to ALC, and almost $45 \%$ of them were for hepatic encephalopathy [85]; thus, the management of this complication at home with the help of a caregiver is of a crucial importance [86]. Indeed, the presence of formal and well-trained caregivers periodically in contact with the patient and his/her family can improve the quality of life of both the patient and the family; this figure also enhances the activity of the health service, reducing the number of accesses to the emergency room or ordinary hospitalization $[87,88]$.

\section{Inpatient Treatment}

ALC decompensation (ascites, variceal bleeding, and hepatic encephalopathy) is the major reason for hospitalization in patients affected by ALC, together with liver transplant rejection or other clinical ALC complications (hepatorenal and hepatopulmonary syndrome, ACLF, and spontaneous bacterial peritonitis) $[35,85]$. It would be better for patients to be admitted to COVID-19 disease-free liver units. In the case of SARS-CoV-2 infection, compensated ALD patients are admitted for inpatient care if the symptomatology is severe and/or additional risk factors are present (diabetes, obesity, cardiovascular pathologies), whereas patients who test positive to SARS-CoV-2 infection with liver decompensation and/or HCC need early admission to accredited facilities. It is appropriate to choose nonhepatotoxic drugs (or those with low hepatotoxicity) and closely monitor possible drug interference with anti-craving drugs, psychotropic drugs, or immunosuppressive medications. Thus, the decision making will be different in relation to the presence of compensated ALD or decompensated ALC (Table 3).

\section{Liver Transplantation}

The possibility of false-negative nasopharyngeal/pharyngeal swabs has been reported in the literature. For this reason, pending containment of the epidemic, it is better to perform liver transplantation (LT) only in the most urgent cases. This is especially true in epidemic or high-risk areas ("red zones"). All recipients on the list must be screened 
Table 3 Management of compensated and decompensated ALC in AUD patients in the COVID-19 era

\section{Compensated ALC}

\section{No SARS-CoV-2 infection}

Use telemedicine for periodic visits (outpatient visits in safety only for patients who resumed heavy alcohol consumption in order to plan a new detoxification program)

Routine laboratory tests to monitor liver function can be performed by request of the primary care physician

Maintain routine ultrasound and endoscopic checks in safety

Do not stop anti-craving therapy, psychotherapy, and attendance at self-help groups (possibly via web)

SARS-CoV-2 infection

If asymptomatic, patients must stay at home, use telemedicine for periodic visits and for a rapid check of the clinical condition and to monitor adherence to therapy

If symptomatic, multi-disciplinary collaboration is needed, patients may need hospitalization in a COVID-19 area, and if he/she needs to enter a clinical pharmacological trial for the treatment of COVID-19 in accordance with specialists in infectious diseases, liver toxicity of drugs may be considered

Discontinuation of adversative (i.e., disulfiram) or anti-craving (i.e., acamprosate) and psychotropic drugs (i.e., tricyclic antidepressants, antipsychotics, antiepileptics, bupropion) currently used to treat patients with AUD and psychiatric co-morbidity needs to be carefully evaluated since the pharmacological therapies used to treat COVID-19 may negatively interfere

\section{Decompensated ALC}

\section{No SARS-CoV-2 infection}

Outpatient or day service visits in safety where check of therapy, paracentesis, routine laboratory tests, ultrasound endoscopic checks in equipped and safe rooms may be performed; routine laboratory tests can be also prescribed by primary care physician

Hospitalization may be necessary for severe hepatic encephalopathy not managed at home, refractory ascites, management of hepatorenal and hepatopulmonary syndromes, variceal bleeding, spontaneous bacterial peritonitis, and a condition of acute on chronic liver failure

Limit the use of anti-craving drugs to acamprosate or baclofen, and in the case of treatment of alcohol withdrawal syndrome use short half-life benzodiazepines (lorazepma and oxazepam) or sodium oxybate

SARS-CoV-2 infection

Hospitalization is needed, and instrumental examinations can be limited to emergencies (i.e., esophago-gastro-duodenoscopy for gastrointestinal bleeding)

In patients with hepatocellular carcinoma, loco-regional therapy should be temporarily postponed until after recovery from the infection, and immune therapy temporarily discontinued

Use corticosteroids only in patients with severe COVID-19 needing oxygen support, and with extreme caution considering the reduced immune response frequently found in AUD patients

Use low molecular weight heparin with extreme caution due to the thrombocytopenia in ALC patients, and considering that the real efficacy of this drug is still unclear

Since remdesivir, hydroxychloroquine, lopinavir, and interferon regimens had little or no effect on hospitalized patients with COVID-19, and since deterioration of liver and kidney function is frequently found during these treatments, these drugs should be avoided in patients with decompensated ALC

\section{Liver transplantation for ALC}

Before LT

Multidisciplinary clinical management (hepatologists, psychologists, and caregivers) of patients may be done using telemedicine with minimal exposure to medical staff

Before LT in both donors and recipients, nasopharyngeal/pharyngeal swab check for COVID-19 should be performed, taking into account that a negative test cannot completely rule out the presence of infection

If a recipient is found to be SARS-CoV-2 positive, LT may be postponed until after recovery from the infection

All SARS-CoV-2-positive donors will be excluded

Living-donor transplantation must be considered on a case-by-case basis

In the case of COVID-19 infection, early admission in accredited facilities is recommended

After LT

Multidisciplinary clinical management of patients may be done using telemedicine with minimal exposure to medical staff

In the case of post-surgical complications or rejection, provide hospitalization in Hepatology or Surgical COVID-19-free area

Emphasize the need for vaccination

Immunosuppression therapy should not be changed; however, mycophenolate withdrawal or temporary conversion to calcineurin inhibitors (tacrolimus or cyclosporine) or mTOR inhibitors (everolimus) until disease resolution could be beneficial in hospitalized patients with COVID-19, while a reduction of therapy with calcineurin and mTOR inhibitors should be considered

In the case of a patient with COVID-19 infection subjected to LT, and included in a COVID-19 clinical trial, avoid drugs interfering with immune-suppressants and, in any case, adjust therapy dosage 
repeatedly at regular intervals (the timing will be defined by the relative center also in relation to the characteristics of the individual recipients) [89].

If the recipient is in an epidemic area and the conditions allow it, the transplant can be delayed. If the patient falls within particular parameters with worsening of liver condition (MELD $\geq 20$, risk of drop-out for HCC) [12, 15, 30, $90,91]$, he/she will undergo a new swab before LT. If a recipient is found to be positive, $\mathrm{LT}$ may be postponed until after recovery from SARS-CoV-2 infection [18]. Only if a potential recipient is symptomatic for SARS-CoV-2, can a computed tomography chest without contrast be performed to look for typical opacities $[18,92]$

If the recipient is not in an epidemic area and has a negative history of symptoms or recent contact with patients with COVID-19, he/she will undergo a nasopharyngeal/pharyngeal swab. Of course, all positive donors will be excluded. All donors need to perform a nasopharyngeal/pharyngeal swab [93].

In post-LT hospitalization, transfers between wards must be limited and visits by relatives to the patient's bedside have to be forbidden. In the post-LT phase, if the patient is negative, immunosuppression will continue with the common dosages; in the case of testing positive to SARS$\mathrm{CoV}-2$ infection, immune-suppression will be adapted due to the pharmacological interactions with the experimental therapies (Table 3) [89, 93]. Moreover, taking into account that the incidence of COVID-19 is higher in liver transplant patients, the correct management of immune-suppression therapy becomes relevant. Complete immune-suppression withdrawal may not be justified $[18,94]$. However, mycophenolate produces a cytostatic effect on activated lymphocytes [95], and the virus SARS-CoV-2 has a direct cytotoxic effect on lymphocytes [96]. Therefore, mycophenolate and SARS-CoV-2 may exert a synergic and deleterious effect on depleting peripheral lymphocytes. In particular, a recent study has shown that mycophenolate at doses higher than $1000 \mathrm{mg} /$ day could increase the risk of severe COVID-19 [94]. Thus, mycophenolate withdrawal or temporary conversion to calcineurin inhibitors (tacrolimus or cyclosporine) or mTOR inhibitors (everolimus) until disease resolution could be beneficial in hospitalized patients. On the other hand, calcineurin and mTOR inhibitors are not associated with worse outcomes in COVID-19; thus, only a reduction of their doses with a frequent checking of their plasma concentrations may be enough $[18,94]$ (Table 3 ).

\section{Group Activities}

Suspension of all group activities such as alcoholic anonymous or groups with operators and patients is warranted. However, AUD is a disease with frequent relapses, and continuous psychosocial support is crucial. In the context of
COVID-19 lockdown, the absence of self-help groups may be a serious problem, also in consideration of the fact that only a small number of self-help groups are active via web.

\section{Conclusions}

AUD/ALD is complicated clinical conditions in which medical and psycho-social problems are combined. The isolation induced by the SARS-CoV-2 pandemic has significantly worsened the suffering of patients with AUD/ALD and of their families as well. HCs will, therefore, need to improve and review their clinical activities practiced up till now, starting from the relationship between operators, patients, and caregivers which plays an important role in the management and in the prevention of relapses and complications of AUD and ALD.

Moreover, all online services need to be up-graded with telemedicine/telehealth or through meetings where the engagement of clinicians, nurses, patients, and caregivers will become of paramount importance [97-100]. However, patients from lower socioeconomic backgrounds who lack access to smartphones or Internet service will be particularly affected, with the exacerbation of social isolation, anxiety, and relapses [100]. Furthermore, behavioral programs (i.e., counseling and alcohol relapse prevention programs) and alcoholic anonymous groups may not be currently available and this may worsen patients' conditions [101]. In addition, misinformation about alcohol and COVID-19 is circulating, with fake news spread through mass-media reports that alcoholic beverages can reduce the risk of contracting COVID19 may increase the risk of harmful drinking [102, 103].

Taking into account that consensus data of the international societies are continuously under review, our statement and recommendations seem to walk in line with some societies' consensus opinions. While the AASLD [16] and the APASL [17] consensus statements have clearly highlighted recommendations for the management of liver diseases in SARS-CoV-2-infected patients with no particular focus on the management of patients with ALD and AUD [16], the EASL with the ESCMID [14, 15], and the WGO [18] have focused attention also on the management of patients with ALD and SARS-CoV-2 infection, finding our position in line particularly on some specific issues: (a) Chronic alcohol consumption may increase an individual's susceptibility to ARDS secondary to SARS-CoV-2 infection, (b) social isolation can lead to new or increased alcohol consumption with an increase in alcohol-related admissions including new liver decompensation, (c) the implementation of telemedicine for patients affected by AUD and ALD.

Thus, the COVID-19 pandemic has rapidly carried us toward a new governance scenario of AUD and ALD which necessarily requires an in-depth review of the 
management of these diseases, with a new safe approach without losing the therapeutic efficacy of multidisciplinary treatment $[98,99]$.

\section{Declarations}

Conflicts of interest We have no disclosures of potential conflicts of interest to declare. Since the manuscript is a POSITION PAPER without the recruitment of humans or animals, informed consent was not necessary.

Ethics and responsibility of authors The manuscript has not been submitted elsewhere. Consent to submit has been received explicitly from all co-authors before the work was submitted. Authors whose names appear on the submission have contributed sufficiently to the scientific work and therefore share collective responsibility and accountability for the results.

\section{References}

1. Zhou F, Yu T, Du R, Fan G, Liu Y et al. Clinical course and risk factors for mortality of adult inpatients with COVID19 in Wuhan, China: a retrospective cohort study. Lancet. 2020;395:1054-1062.

2. Huang C, Wang Y, Li X, Ren L, Zhao J et al. Clinical features of patients infected with 2019 novel coronavirus in Wuhan China. Lancet. 2020;395:497-506.

3. Wiersinga WJ, Rhodes A, Cheng AC, Peacock SJ, Prescott HC. Pathophysiology, transmission, diagnosis, and treatment of coronavirus disease 2019 (COVID-19) A Review. JAMA. 2019;1:1. https://doi.org/10.1001/jama.2020.12839.

4. World Health Organization. Global status report on alcohol and health. 2018.

5. GBD. Alcohol collaborators alcohol use and burden for 195 countries and territories, 1990-2016: a systematic analysis for the global burden of disease study 2016. Lancet. 2018;392:1015-1035.

6. Carvalho AF, Heilig M, Perez A, Porbst C, Rhem J. Alcohol use disorders. Lancet. 2019;394:781-792.

7. American Psychiatric Association. Diagnostic and statistical manual of mental disorders, 5th edn. Washington: American Psychiatric Publishing; 2013.

8. Grant BF, Goldstein RB, Saha TD et al. Epidemiology of DSM-5 alcohol use disorder. JAMA Psychiatry. 2015;72:757-766

9. Clay JM, Parker MO. Alcohol use and misuse during the COVID19 pandemic: a potential public health crisis? Lancet Public Health. 2020;5:e259. https://doi.org/10.1016/S2468-2667(20) 30088-8.

10. Zahir A, Oli A, Zhou A, Hanbin S et al. Epidemic of COVID-19 in China and associated psychological problems. Asian J Psychiatr. 2020;51:102092. https://doi.org/10.1016/j.ajp.2020.102092.

11. Connor JP, Haber PS, Hall WD. Alcohol use disorders. Lancet. 2015;387:988-998.

12. European Association for the Study of the Liver. EASL clinical practice guidelines: management of alcohol-related liver disease. J Hepatol. 2018;69:154-181.

13. Pimpin L, Cortez-Pinto H, Negro F et al. Burden of liver disease in Europe: epidemiology and analysis of risk factors to identify prevention policies. J Hepatol. 2018;69:718-735.
14. Boettler T, Newsome PN, Mondelli MU et al. Care of patients with liver disease during the COVID-19 pandemic: EASLESCMID position paper. JHEP Rep. 2020;2:100-113.

15. Boettler T, Marjot T, Newsome PN, Mondelli MU, Maticic $\mathrm{M}$ et al. Impact of COVID-19 on the care of patients with liver disease: EASL-ESCMID position paper after 6 months of the pandemic. J HEP Rep. 2020;2:100169. https://doi.org/ 10.1016/j.jhepr.2020.100169.

16. Fix OK, Hameed B, Fontana RJ, Kwok RM, McGuire BM et al. Clinical best practice advice for hepatology and liver transplant providers during the COVID-19 pandemic: AASLD expert panel consensus statement. Hepatology. 2020;72:287-304.

17. Wong GL, Wong VW, Thompson A, Jia J, Hou J et al. Management of patients with liver derangement during the COVID-19 pandemic: an Asia-Pacific position statement. Lancet Gastroenterol Hepatol. 2020;5:776-787.

18. Hamid S, Alvares da Silva MR, Burak KW, Chen T et al. J Clin Gastroenterol. 2021;55:1-11.

19. European Centre for Disease Prevention and Control, 2020 https://www.ecdc.europa.eu/en/covid-19-pandemic.

20. World Health Organization, 2020: https://www.who.int/emerg encies/diseases/novel-coronavirus-2019.

21. Chinese Center for Disease Control and Prevention (China CDC), 2020: http://www.chinacdc.cn/en/COVID19/.

22. Zaki AM, van Boheemen S, Bestebroer TM, Osterhaus ADME, Fouchier RAM. Isolation of a novel coronavirus from a man with pneumonia in Saudi Arabia. $N$ Engl J Med. 2012;367:1814-1820.

23. Peiris JS, Yuen KY, Osterhaus AD et al. The severe acute respiratory syndrome. N Engl J Med. 2003;349:2431-2441.

24. Jiang F, Deng L, Zhang L, Stor K. Review of the clinical characteristics of Coronavirus Disease 2019 (COVID-19). J Gen Intern Med. 2020;35:1545-1549.

25. Gu J, Han B, Wang J. COVID-19: gastrointestinal manifestations and potential fecal-oral transmission. Gastroenterology. 2020;158:1518-1519.

26. Gao QY, Chen YX, Fang JY. 2019 novel coronavirus infection and gastrointestinal tract. J Dig Dis. 2020;21:125-126.

27. Mehta P, McAuley DF, Brown M et al. Covid-19: consider cytokine storm syndromes and immunosuppression. Lancet. 2020;395:1033-1034.

28. Kopel J, Perisetti A, Gajendran M, Boregowda U, Goyal H. Clinical insights into the gastrointestinal manifestations of COVID19. Dig Dis Sci. 2020;65:1932-1939.

29. Ahn DG, Shin HJ, Kim MH et al. Current status of epidemiology, diagnosis, therapeutics, and vaccines for novel coronavirus disease 2019 (Covid-19). J Microbiol Biotechnol. 2020;30:313-324.

30. Seitz HK, Bataller R, Cortez-Pinto $\mathrm{H}$ et al. Alcoholic liver disease. Nat Rev Dis Primers. 2018;4:16.

31. Testino G, Vignoli T, Patussi V, Scafato E, Caputo F. SIA board (Appendix A) and the external expert supervisors (Appendix B) Management of end-stage alcohol-related liver disease and severe acute alcohol-related hepatitis: position paper of the Italian Society on Alcohol (SIA). Dig Liver Dis. 2020;52:21-32.

32. Sidhu SS, Goyal O, Kishore H, Sidhu S. New paradigms in management of alcoholic hepatitis: a review. Hepatol Int. 2017;11:255-267.

33. Roth NC, Qin J. Histopathology of alcohol related liver disease. Clin Liver Dis. 2019;23:11-23.

34. Bajaj JS, Moreau R, Kamath PS et al. Acute on chronic liver failure: getting ready for prime-time. Hepatology. 2018;68:1621-1632.

35. European Association for the Study of the Liver. European association for the study of the liver EASL clinical practice guidelines for the management of patients with decompensated cirrhosis. J Hepatol. 2018;69:406-460. 
36. Qi F, Qian S, Zhang S, Zhang Z. Single cell RNA sequencing of 13 human tissues identify cell types and receptors of human coronaviruses. Biochem Biophys Res Commun. 2020;526:135-140.

37. Okuno F, Arai M, Ishii $\mathrm{H}$ et al. Mild but prolonged elevation of serum angiotensin converting enzyme (ACE) activity in alcoholics. Alcohol. 1986;3:357-359.

38. Xu L, Liu J, Lu M et al. Liver injury during highly pathogenic human coronavirus infections. Liver Int. 2020;40:998-1004.

39. Jothimani D, Venugopal R, Abedin MF, Kaliamoorthy I, Rela M. COVID-19 and the liver. J Hepatol. 2020;73:1231-1240.

40. Zhang C, Shi L, Wang FS. Liver injury in COVID-19: management and challenges. Lancet Gastroenterol Hepatol. 2020;5:428-430.

41. Surveillance Epidemiology of Coronavirus (COVID-19) Under Research Exclusion. SECURE-Cirrhosis Registry. Available at: https://covidcirrhosis.web.unc.edu/. Accessed April 25, 2020.

42. Wu Y, Li H, Guo X, Yoshida EM, Mendez-Sanchez N et al. Incidence, risk factors, and prognosis of abnormal liver biochemical tests in COVID-19 patients: a systematic review and meta-analysis. Hepatol Int. 2020;14:621-637.

43. AASLD. AASLD Acts on COVID-19 and Its Impact on Liver Patients - AASLD News. Available at: https://www.aasldnews. org/aasld-acts-on-covid-19-and-its-impact-on-liver-patients/. Accessed April 13, 2020.

44. Da BL, Im GY, Schiano TD. COVID-19 hangover: a rising tide of alcohol use disorder and alcohol-associated liver disease. Hepatology. 2020. https://doi.org/10.1002/hep.31307.

45. Rosenberg ES, Dufort EM, Udo T et al. Association of treatment with hydroxychloroquine or azithromycin with in-hospital mortality in patients with COVID-19 in New York state. JAMA. 2020;323:2493-2502.

46. Dong L, Hu S, Gao J. Discovering drugs to treat coronavirus disease 2019 (COVID-2019). Drug Discov Ther. 2020;19:149-150.

47. Barlow A, Landolf KM, Barlow B et al. Review of emerging pharmacotherapy for the treatment of coronavirus disease 2019. Pharmacother. 2020;40:416-437.

48. Garrido I, Liberal R, Macedo G. Review article: COVID-19 and liver disease-what we know on 1st May 2020. Alim Pharmacol Ther. 2020;52:267-275.

49. Pan H, Peto R, Henao-Restrepo AM, Preziosi MP, Sathiyamoorthy $\mathrm{V}$ et al. Repurposed antiviral drugs for covid-19 - interim WHO solidarity trial results WHO solidarity trial consortium. $N$ Engl J Med. 2020. https://doi.org/10.1056/NEJMoa2023184.

50. Center for Drug Evaluation and Research. Combined crossdiscipline team leader, division director, and ODE director summary review for NDA 214787. October 21, 2020 (https://www.accessdata.fda .gov/drugsatfda_docs/nda/2020/ 214787Orig1s000Sumr.pdf).

51. https://www.aifa.gov.it/aggiornamento-sui-farmaci-utilizzabiliper-il-trattamento-della-malattia-covid19

52. Ahmed MH, Hassan A. Dexamethasone for the treatment of coronavirus disease (COVID-19): a review. SN Compr Clin Med. 2020;31:1-10.

53. Cusinato J, Cau Y, Calvani AM, Mori M. Repurposing drugs for the management of COVID-19. Expert Opin Ther Pat. 2020. https://doi.org/10.1080/13543776.2021.1861248.

54. Sulkowski MS. Drug-induced liver injury associated with antiretroviral therapy that includes HIV-1 protease inhibitors. Clin Infect Dis. 2004;38:S90-S97.

55. Fries JF, Singh G, Lenert L, Furst DE. Aspirin, hydroxychloroquine, and hepatic enzyme abnormalities with methotrexate in rheumatoid arthritis. Arthritis Rheum. 1990;33:1611-1619.

56. Meyerowitz EA, Vannier AGL, Friesen MGN et al. Rethinking the role of hydroxychloroquine in the treatment of COVID-19. FASEB J. 2020;34:6027-6037.
57. Genovese MC, Kremer JM, van Vollenhoven RF et al. Transaminase levels and hepatic events during tocilizumab treatment: pooled analysis of long-term clinical trial safety data in rheumatoid arthritis. Arthritis Rheumatol. 2017;69:1751-1761.

58. Guzzo CA, Furtek CI, Porras AG et al. Safety, tolerability, and pharmacokinetics of escalating high doses of ivermectin in healthy adult subjects. J Clin Pharmacol. 2002;42:1122-1133.

59. Grein J, Ohmagari N, Shin D et al. Compassionate use of remdesivir for patients with severe Covid-19. $N$ Engl J Med. 2020;382:2327-2336.

60. Irie K, Nakagawa A, Fujita $\mathrm{H}$ et al. Pharmacokinetics of favipiravir in critically Ill patients with COVID-19. Clin Transl Sci. 2020. https://doi.org/10.1111/cts.12827.

61. Antonelli M, Ferrulli A, Sestito L et al. Alcohol addiction - the safety of available approved treatment options. Exp Op Drug Saf. 2018;17:169-177.

62. Caputo F, Vignoli T, Grignaschi A et al. Pharmacological management of alcohol dependence: from mono-therapy to pharmacogenetics and beyond. Eur Neuropsychopharmacol. 2014;24:181-191.

63. Mutschler J, Grosshans M, Soyka M et al. Current findings and mechanisms of action of disulfiram in the treatment of alcohol dependence. Pharmacopsychiatry. 2016;49:137-141.

64. Aubin HJ, Daeppen JB. Emerging pharmacotherapies for alcohol dependence: a systematic review focusing on reduction in consumption. Drug Alcohol Depend. 2013;133:15-29.

65. Oka M, Hirouchi M, Tamura M et al. Acamprosate monocalcium bis (3-acetamidopropane-1-sulfonate) reduces ethanoldrinking behavior in rats and glutamate-induced toxicity in ethanol-exposed primary rat cortical neuronal cultures. Eur $J$ Pharmacol. 2013;718:323-331.

66. Plosker GL. Acamprosate: a review of its use in alcohol dependence. Drugs. 2015;75:1255-1268.

67. Mann K, Kiefer F, Spanagel R et al. Acamprosate: recent findings and future research directions. Alcohol Clin Exp Res. 2008;32:1105-1110.

68. Umhau JC, Momenan R, Schwandt ML et al. Effect of acamprosate on magnetic resonance spectroscopy measures of central glutamate in detoxified alcohol-dependent individuals: a randomized controlled experimental medicine study. Arch Gen Psychiatry. 2010;67:1069-1077.

69. Addolorato G, Lesch OM, Maremmani I et al. Post-marketing and clinical safety experience with sodium oxybate for the treatment of alcohol withdrawal syndrome and maintenance of abstinence in alcohol-dependent subjects. Expert Opin Drug Saf. 2020;19:159-166.

70. Castillo-Carniglia A, Keyes KM, Hasin DS, Cerdá M. Psychiatric comorbidities in alcohol use disorder. Lancet Psychiatry. 2019;6:1068-1080.

71. Sun J, Deng X, Chen X et al. Incidence of adverse drug reactions in Covid-19 patients in China: an active monitoring study by hospital pharmacovigilance system. Clin Pharmacol Ther. 2020. https://doi.org/10.1002/cpt.1866.

72. Guerzoni S, Pellesi L, Pini LA, Caputo F. Drug-drug interactions in the treatment for alcohol use disorders: a comprehensive review. Pharmacol Res. 2018;133:65-76.

73. Chen CY, Wang FL, Lin CC. Chronic hydroxychloroquine use associated with QT prolongation and refractory ventricular arrhythmia. Clin Toxicol (Phila). 2006;44:173-175.

74. Mercuro NJ, Yen CF, Shim DJ et al. Risk of QT interval prolongation associated with use of hydroxychloroquine with or without concomitant azithromycin among hospitalized patients testing positive for coronavirus disease 2019 (COVID-19). JAMA Cardiol. 2020. https://doi.org/10.1001/jamacardio.2020. 1834. 
75. Beach SR, Celano CM, Sugrue AM et al. QT prolongation, torsades de pointes, and psychotropic medications: a 5-year update. Psychosomatics. 2018;59:105-122.

76. Gartlehner G, Thieda P, Hansen RA et al. Comparative risk for harms of second generation antidepressants: a systematic review and meta-analysis. Drug Saf. 2008;31:851-865.

77. Voican CS, Corruble E, Naveau S, Perlemuter G. Antidepressantinduced liver injury: a review for clinicians. Am J Psychiatry. 2014;171:404-415.

78. Kaner EF, Beyer FR, Garnett $\mathrm{C}$ et al. Personalized digital interventions for reducing hazardous and harmful alcohol consumption in community-dwelling populations. Cochrane Database Syst Rev. 2017;9:CD011479.

79. Molfenter T, Brown R, O’Neill A, Kopetsky E, Toy A. Use of telemedicine in addiction treatment: current practices and organizational implementation characteristics. Int J Telemed Appl. 2018. https://doi.org/10.1155/2018/3932643.

80. Hollander JE, Carr BG. Virtually perfect? Telemedicine for covid-19. N Engl J Med. 2020;382:1679-1681.

81. Serper M, Cubell AW, Deleener ME, Casher TK, Rosenberg DJ, Whitebloom D, Rosin RM. Telemedicine in liver disease and beyond: can the COVID-19 crisis lead to action? Hepatology 2020;1:1. https://doi.org/10.1002/hep.31276.

82. Kruse CS, Karem P, Shifflett K, Vegi L, Ravi K, Brooks M. Evaluating barriers to adopting telemedicine worldwide: a systematic review. J Telemed Telecare. 2018;24:4-12.

83. Medicare Telemedicine Health Care Provider Fact Sheet. Centers for Medicare \& Medicaid Services. Available at: https://www. cms.gov/newsroom/fact-sheets/medicare-telemedicine-healthcare-provider-fact-sheet.

84. Italian Government, Official Bulletin, May $18^{\text {th }} 2020$ : https:// www.gazzettaufficiale.it/eli/id/2020/05/18/20A02727/sg.

85. Gaspar R, Rodrigues S, Silva M et al. Predictive models of mortality and hospital readmission of patients with decompensated liver cirrhosis. Dig Liver Dis. 2019;51:1423-1429.

86. Balbinot $\mathrm{P}$, Leone $\mathrm{S}$, Testino $\mathrm{G}$, Caputo F. Hospital readmission of patients with hepatic encephalopathy: Is the introduction of the formal caregiver useful in care management? Dig Liver Dis. 2020;52:358-359.

87. Aspinall RJ. Reducing recurrent hospital admissions in patients with decompen-sated cirrhosis. Br J Hosp Med. 2018;79:93-96.

88. Montagnese S, Amato E, Schiff S et al. A patients' and caregivers' perspective on hepatic hencephalopathy. Metab Brain Dis. 2012;27:567-572.

89. Gori A, Dondossala D, Antonelli B et al. Coronavirus disease 2019 and transplantation: a view from the inside. Am J Transplant. 2020. https://doi.org/10.1111/AJT.15853.

90. Testino G, Vignoli T, Patussi V, Scafato E, Caputo F. Liver transplantation for patients with and-stage alcohol-related liver disease and severe acute alcohol-related hepatitis: a brief update. Alcologia. 2020;39:48-54.
91. Mathurin P, Lucey MR. Liver transplantation in patients with alcohol-related liver disease: current status and future directions. Lancet Gastroenterol Hepatol. 2020;5:507-514.

92. American Society of Transplantation. 2019-nCoV (Coronavirus): FAQs for Organ Transplantation. www.notifylibrary.org/ sites/default/files/COVID19\%20FAQ\%20Tx\%20Centers\%2005. 11.2020.pdf

93. Maggi U, De Carlis L, Daniel Y et al. The impact of the Covid-19 outbreak on liver transplantation programmes in Northern Italy. Am J Transplant. 2020;20:1840-1848.

94. Colmenero J, Rodríguez-Perálvarez M, Salcedo M, Arias-Milla A, Muñoz-Serrano A et al. Epidemiological pattern, incidence, and outcomes of COVID-19 in liver transplant patients. J Hepatol. 2021;74:148-155.

95. Allison AC, Eugui EM. Mycophenolate mofetil and its mechanisms of action. Immunopharmacology. 2000;47:85-118.

96. Qin C, Zhou L, Hu Z, Zhang S, Yang S et al. Dysregulation of immune response in patients with coronavirus 2019 (COVID-19) in Wuhan China. Clin Infect Dis. 2020;71:762-768.

97. Kruse CS, Lee K, Watson JB et al. Measures of effectiveness, efficiency and quality of telemedicine in the management of alcohol abuse, addiction, and rehabilitation: systematic review. J Med Internet Res. 2020;22:e13252.

98. Bottaro LC, Leone S, Sampietro L et al. Management of addiction medicine: sharing medicine? Minerva Med. 2020;111:1-3.

99. Testino G, Pellicano R. Alcohol consumption in the Covid-19 Era. Minerva Gastroenterol Dietol. 2020;66:90-92.

100. Simonetto DA, Shah VH, Kamath PS. Outpatient management of alcohol-related liver disease. Lancet Gastroenterol Hepatol. 2020;5:485-493.

101. Hoffman J With Meetings Banned, Millions Struggle to Stay Sober On Their Own. New York Times. Available at: https:// www.nytimes.com/2020/03/26/health/coronavirus-alcoholicsdrugs-online.html.

102. False Claim: Saint Luke's Hospital Notice Advises Consuming Alcoholic Beverages to Reduce Coronavirus Risk. Reuters. Available at: https://www.reuters.com/article/uk-factcheck-hospi tal-consume-alcohol-co/false-claim-saint-lukes-hospital-noticeadvises-consuming-alcoholic-beverages-to-reduce-coronavirusrisk-idUSKBN21J6W2.

103. Black H, Gill J, Chick J. The price of a drink: levels of consumption and price paid per unit of alcohol by Edinburgh's ill drinkers with a comparison to wider alcohol sales in Scotland. Addiction. 2011;106:729-736.

Publisher's Note Springer Nature remains neutral with regard to jurisdictional claims in published maps and institutional affiliations. 


\section{Authors and Affiliations}

Gianni Testino ${ }^{1} \cdot$ Teo Vignoli $^{2}$. Valentino Patussi ${ }^{3} \cdot$ Pierluigi Allosio $^{4} \cdot$ Maria Francesca Amendola $^{5}$. Sarino Aricò ${ }^{6}$. Aniello Baselice ${ }^{7}$ - Patrizia Balbinot ${ }^{1} \cdot$ Vito Campanile $^{8} \cdot$ Tiziana Fanucchi $^{3} \cdot$ Giovanni Greco $^{9} \cdot$ Livia Macciò $^{10}$. Cristina Meneguzzi ${ }^{11}$. Davide Mioni ${ }^{12}$. Vincenzo Ostilio Palmieri ${ }^{13} \cdot$ Michele Parisi $^{14}$. Doda Renzetti ${ }^{15}$. Raffaella Rossin ${ }^{16}$. Claudia Gandin ${ }^{17}$. Luigi Carlo Bottaro ${ }^{18}$. Mauro Bernardi ${ }^{19}$. Giovanni Addolorato ${ }^{20}$. Lisa Lungaro ${ }^{21}$. Giorgio Zoli ${ }^{21,22,23}$. Emanuele Scafato ${ }^{17}$ • Fabio Caputo ${ }^{21,22,23}$ (1)

1 Unit of Addiction and Hepatology, Regional Centre On Alcohol, ASL3 San Martino Hospital, Genova, Italy

2 Unit of Addiction Treatment, Lugo, RA, Italy

3 Regional Centre On Alcohol, Careggi Hospital, Firenze, Italy

4 Alcohol Unit, Torino, Italy

5 Alcohol Unit, Cosenza, Italy

6 Gastroenterology Unit, Mauriziano Hospital, Torino, Italy

7 Alcohol Unit, Salerno, Italy

8 Alcohol Unit, Bari, Italy

9 Alcohol Unit, Ravenna, Italy

10 Alcoholo Unit, Savona, Italy

11 Alcohol Unit, Pordenone, Italy

12 Nursing Home Parco Dei Tigli, Teolo, PD, Italy

13 "Murri" Clinic of Internal Medicine, Department of Biomedical Science and Human Oncology, University of Bari, Bari, Italy

14 Alcohol Unit, Nicosia, EN, Italy
15 Department of Internal Medicine, Mater Dei Hospital, Bari, Italy

16 Alcohol Unit, Milano, Italy

17 National Observatory On Alcohol, National Institute of Health, Roma, Italy

18 Local Health Service, Liguria, Italy

19 Department of Medical and Surgical Sciences, University of Bologna, Bologna, Italy

20 Alcohol Use Disorder and Alcohol Related Disease Unit, Department of Internal Medicine and Gastroenterology, Fondazione Policlinico Universitario A. Gemelli IRCCS, Rome, Italy

21 Department of Translational Medicine, University of Ferrara, Ferrara, Italy

22 Department of Internal Medicine, SS Annunziata Hospital, University of Ferrara, Via Vicini 2, 44042 Cento, FE, Italy

23 Centre for the Study and Treatment of Alcohol-Related Diseases, Department of Translational Medicine, University of Ferrara, Ferrara, Italy 